\title{
Digital Geometry from a Geometric Algebra Perspective
}

\author{
Lilian Aveneau, Laurent Fuchs, and Eric Andres \\ Laboratoire XLIM-SIC UMR CNRS 7252, Université de Poitiers, \\ Bld Marie et Pierre Curie, BP 30179, 86962, Futuroscope Chasseneuil Cedex, France \\ \{lilian.aveneau, laurent.fuchs, eric.andres\}@univ-poitiers.fr
}

\begin{abstract}
To model Euclidean spaces in computerized geometric calculations, the Geometric Algebra framework is becoming popular in computer vision, image analysis, etc. Focusing on the Conformal Geometric Algebra, the claim of the paper is that this framework is useful in digital geometry too. To illustrate this, this paper shows how the Conformal Geometric Algebra allow to simplify the description of digital objects, such as k-dimensional circles in any n-dimensional discrete space. Moreover, the notion of duality is an inherent part of the Geometric Algebra. This is particularly useful since many algorithms are based on this notion in digital geometry. We illustrate this important aspect with the definition of k-dimensional spheres.
\end{abstract}

Keywords: Digital Geometry, Geometric Algebra, Conformal Model, Digital Object.

\section{Introduction}

The purpose of this paper is to introduce the computational and mathematical framework of Geometric Algebra (GA) in digital geometry. GA form a powerful mathematical language for expressing and representing geometric objects, transformations or even for working in dual spaces [10 176]. GA are becoming popular in various computer imagery sub-fields such as computer vision or image analysis, and even more largely in fields like physics and engineering [15 517]. The reason for such a popularity is that the mathematical framework of GA is well adapted for handling geometric data of any dimension in a very intuitive way.

GA represent a natural extension of complex numbers and quaternions in arbitrary dimension. Each instance of GA is an associative algebra (known as Clifford algebra) of a real vector space equipped with a given quadratic form. In this paper, we consider the Conformal Geometric Algebra (CGA), defined over the Minkowski space $\mathbb{R}^{n+1,1}$. It offers a very natural representation for circles and spheres (points, lines and planes are simply particular cases of the former) and extension of these geometric primitives in any dimension. CGA also provides a way to represent transformations such as translations, reflections or rotations. 
In Section 2, we present the conformal geometric algebra and some general results on geometric algebra. This is intended as an introduction to CGA for the readers that are not familiar with GA. In Section 3, we apply CGA to define discrete primitives in any dimension. First, based on a previous work 4, discrete hyperspheres and hyperplanes are presented as they often play a special role in algorithms and definitions. The more general case of discrete $k$-sphere (discrete spheres of dimension $k$ in a $n$-dimensional space) is also introduced. Discrete lines, planes and more generally discrete $k$-flats are particular cases of discrete $k$-spheres. Finally, Section 4 proposes a conclusion and some perspectives: One of the hopes in the future, is that generation and recognition algorithms of circles and lines can be somewhat unified in such a general framework.

\section{An Overview of Geometric Algebra}

The basic idea of GA is to use vector subspaces which can be geometrically interpreted as Euclidean geometric primitives, and manipulated with some transformations. Thus, in any dimension, geometric primitives (lines, circles, planes, spheres) and their transformations are represented by vector subspaces.

In this paper we use the conformal model of GA, usually denoted as Conformal Geometric Algebra. The following presentation is mainly based on the book of L. Dorst et al. 6] which provides an accessible and deep description of GA. For a shorter introduction, the reader can consult [12].

\subsection{Building the Conformal Geometric Algebra}

The Vector Space Structure. The starting point to build the CGA is a Euclidean space $\mathbb{R}^{n}$ of dimension $n$ with an orthonormal basis $\left\{e_{1}, e_{2}, \ldots, e_{n}\right\}$. This Euclidean space is naturally equipped with a scalar product such that $e_{i}^{2}=1$ for $i \in\{1,2, \ldots, n\}$.

This Euclidean space is extended with two extra basis vectors $e_{+}$and $e_{-}$ such that $e_{+}{ }^{2}=1$ and $e_{-}^{2}=-1$, and such that $\left\{e_{1}, e_{2}, \ldots, e_{n}, e_{+}, e_{-}\right\}$is an orthogonal basis. This gives the conformal space $\mathbb{R}^{n+1,1}$. Due to its particular scalar product, this space has a particular metric, which is a key point to obtain an interpretation of its vector subspaces as geometric primitives and geometric transformations.

In the rest of this paper, for simplicity, we use the basis $\left\{n_{o}, e_{1}, e_{2}, \ldots, e_{n}, n_{\infty}\right\}$ where $n_{o}=\frac{1}{2}\left(e_{+}+e_{-}\right)$and $n_{\infty}=e_{-}-e_{+}$.

Introducing the Outer Product. Starting from this conformal space, the CGA is built using the GRASSMANN or exterior or outer product, denoted by " $\wedge$ ". Among others properties, the outer product is anticommutative, meaning that for two vector $a$ and $b$, we have $a \wedge b=-b \wedge a$ and $a \wedge a=0$. This product generates new elements from the vectors of $\mathbb{R}^{n+1,1}$. For example, the outer product $a \wedge b$ of two independent vectors $a$ and $b$ is a new element, called a 2 -vector, which lies in a new vector space. The outer product $a \wedge b \wedge c$ of three 
independent vectors $a, b$ and $c$ generates again a vector in a new vector space, and so on until the $(n+2)$-vector space.

Considering the linear combinations of such element:1, we obtain the algebra of vector subspaces of $\mathbb{R}^{n+1,1}$. This algebra $\bigwedge\left(\mathbb{R}^{n+1,1}\right)$ is a graded algebra:

$$
\bigwedge\left(\mathbb{R}^{n+1,1}\right)=\bigwedge^{0}\left(\mathbb{R}^{n+1,1}\right) \oplus \bigwedge^{1}\left(\mathbb{R}^{n+1,1}\right) \oplus \cdots \oplus \bigwedge^{n+2}\left(\mathbb{R}^{n+1,1}\right)
$$

where $\bigwedge^{k}\left(\mathbb{R}^{n+1,1}\right)$ is the vector space of $k$-vectors. The dimension of each of such subspaces is $\left(\begin{array}{c}n+2 \\ k\end{array}\right)$, and then the dimension of the algebra is $2^{n+2}$. The space $\bigwedge^{0}\left(\mathbb{R}^{n+1,1}\right)$ is of dimension 1 and corresponds to the space of the scalars. The space $\bigwedge^{n+2}\left(\mathbb{R}^{n+1,1}\right)$ is the space of pseudo-scalars; it is also a space of dimension 1 spanned by the pseudo-scalar $I_{n+1,1}=n_{o} \wedge \mathbf{I}_{n} \wedge n_{\infty}$ where $\mathbf{I}_{n}=e_{1} \wedge$ $\cdots \wedge e_{n}$ is called the Euclidean pseudo-scalar. Hence, by duality, $\wedge^{n+2}\left(\mathbb{R}^{n+1,1}\right)$ is isomorphic to the scalar space. This principle is extended to each $k$-vector space, which is by duality isomorphic to the $(n+2-k)$-vector space.

Elements of the $k$-vector space $\Lambda^{k}\left(\mathbb{R}^{n+1,1}\right)$ are called multivectors. For a multivector $A$, the part in $\bigwedge^{k}\left(\mathbb{R}^{n+1,1}\right)$ is denoted by $\langle A\rangle_{k}$ and is called the part of grade $k$ of $A$.

Elements that can be written as a product $a_{1} \wedge a_{2} \wedge \cdots \wedge a_{k}$ of 1-vectors are called $k$-blades 2 and are of special interest because they can be interpreted as geometric primitives. These elements represent the vector subspaces of the conformal space $\mathbb{R}^{n+1,1}$, since the outer product of $k$ independent vectors spans a $k$-dimensional vector subspace. Hence, for a particular vector subspace $\mathcal{A}$ of dimension $k$, generated by a $k$-blade $A=a_{1} \wedge \cdots \wedge a_{k}$, we can determine a $k$-blade $I$ such that $A=\lambda I$. The real coefficient $\lambda$ is the (relative) weight of $A$ to the chosen $k$-blade $I$. The attitude of $A$ is the equivalence class $\lambda A$ for any $\lambda \in \mathbb{R}$ and the (relative) orientation of $A$ is the sign of $\lambda$. These three quantities are well known for a vector line; for any vector line defined by a vector $a$ we can define a (unit) vector $i$ such that $a=\lambda i$. In that case the attitude corresponds to the direction of the line.

Introducing the Geometric Product. The geometric product is first defined for two vectors $a$ and $b, a b=a \cdot b+a \wedge b$ where "." is the scalar product of $\mathbb{R}^{n+1,1}$. The geometric product has no symbol to denote it. This product is then linearly extended to any algebra element using the following properties for all scalars $\alpha$ and multivectors $A, B$ and $C$ :

$$
\begin{array}{ll}
1 A=A 1=A, & A(B+C)=A B+A C, \quad(B+C) A=B A+C A, \\
(A B) C=A(B C), & (\alpha A) B=A(\alpha B)=\alpha(A B) .
\end{array}
$$

\footnotetext{
${ }^{1}$ Recall that an algebra is a vector subspace equipped with a product. Hence, addition of vector subspaces and scalar multiplication of vector subspaces are available operations.

${ }^{2}$ In some textbooks, they are also called decomposable $k$-vectors.
} 
This product is the fundamental product of the Geometric Algebra, the other products can be defined from it. The definitions of the previous outer product and the left contraction used below from the geometric product are:

outer product : $A_{k} \wedge B_{l}=\left\langle A_{k} B_{l}\right\rangle_{k+l}$ left contraction : $\left.A_{k}\right\rfloor B_{l}=\left\langle A_{k} B_{l}\right\rangle_{l-k}$ where the indexes denote the grade of the multivectors $A$ and $B$.

The most important notion introduced by the geometric product is the possibility to compute an inverse of a $k$-blade that has nonzero norm. For example, the inverse of $I_{n+1,1}$ is given by $I_{n+1,1}^{-1}=n_{o} \wedge \mathbf{I}_{n}^{-1} \wedge n_{\infty}$ where $\mathbf{I}_{n}^{-1}=(-1)^{n(n-1) / 2} \mathbf{I}_{n}$. This lets us define the dual of a multivector $A$ as $A^{*}=A I_{n+1,1}^{-1}$, or equivalently $\left.A^{*}=A\right\rfloor I_{n+1,1}^{-1}$. If $A_{k}$ is a $k$-blade then $A_{k}^{*}$ is an $(n+2-k)$-blade which represents the orthogonal complement 3 of the $k$-blade $A_{k}$.

Euclidean Point Representation in CGA. Any Euclidean point $\mathbf{p}$ of $\mathbb{R}^{n}$ is represented by a vector $p$ of the conformal space $\mathbb{R}^{n+1,1}$ by:

$$
p=F(\mathbf{p})=n_{o}+\mathbf{p}+\frac{1}{2} \mathbf{p}^{2} n_{\infty} .
$$

This vector $p$ is normalized as the coefficient of $n_{o}$ is 1 . Else, it can be normalized using $\frac{p}{-n_{\infty} \cdot p}$. In a general setting, the coefficient of $n_{o}$ is the weight of the vector $p$ and, using $n_{o} \cdot n_{\infty}=-1$, is equal to $-n_{\infty} \cdot p$.

The dot product of two vectors $p$ and $q$ representing two Euclidean points $\mathbf{p}$ and $\mathbf{q}$ is directly linked to their Euclidean distance $d_{2}(\mathbf{p}, \mathbf{q})$ :

$$
p \cdot q=-\frac{1}{2}(\mathbf{p}-\mathbf{q})^{2}
$$

Hence, for a vector $p \in \mathbb{R}^{n+1,1}$ representing a Euclidean point $\mathbf{p}$, it follows $p \cdot p=0$ and $-n_{\infty} \cdot p \neq 0$; so, Euclidean points are represented by null vectors (i.e. vectors that square to zero). Moreover, the normalization condition and the equality $n_{\infty} \cdot n_{\infty}=0$ tell us that $n_{\infty}$ is a point and can be geometrically interpreted as the point at infinity. The vector $n_{o}$ is also a null vector, so it represents a point and can be geometrically interpreted as the point at the origin in the chosen representation 4 of Euclidean points in the conformal space.

\subsection{Representing Geometric Elements}

Hyperspheres and Hyperplanes. The equation (1) immediately gives us the equation of a hypersphere with the Euclidean point $\mathbf{c}$ as center and radius $\rho$. For a Euclidean point $\mathbf{x}$ of the hypersphere we have:

$$
x \cdot c=-\frac{1}{2} \rho^{2}
$$

\footnotetext{
3 This orthogonality notion refers to the particular metric we have defined on $\mathbb{R}^{n+1,1}$.

${ }^{4}$ Actually any other finite point $p$ can be chosen as point at origin. Since the normalization condition imposes $-n_{\infty} \cdot p=1$, it has the same relation with $n_{\infty}$ as $n_{o}$.
} 
where $x$ and $c$ are the vectors representing the points $\mathbf{c}$ and $\mathbf{x}$. This last equation is equivalent to:

$$
x \cdot\left(c-\frac{1}{2} \rho^{2} n_{\infty}\right)=0
$$

by using the normalizing condition $-n_{\infty} \cdot x=1$. The vector $\sigma=c-\frac{1}{2} \rho^{2} n_{\infty}$ represents the hypersphere with center $\mathbf{c}$ and radius $\rho$.

As the defining equation of a hypersphere is $x \cdot \sigma=0$, we say that the vector $\sigma$ dually represents the hypersphere. From this, a direct representation of a hypersphere can be deduced (see [6]). In brief, in dual representation a hypersphere is represented by a vector which is a 1-dimension vector subspace. Then, taking the dual in $\mathbb{R}^{n+1,1}$ of this vector in leads to a vector subspace $\Sigma$ of dimension $n+1$. This vector subspace is spanned by $n+1$ vectors that can be chosen as representing vectors of $n+1$ Euclidean points. So we can write $\Sigma=p_{1} \wedge p_{2} \wedge \cdots \wedge p_{n+1}$ and $\Sigma^{*}=\sigma$. This means that a Euclidean hypersphere in $\mathbb{R}^{n}$ is defined by $n+1$ Euclidean points.

Considering equation (2) for a Euclidean point $\mathrm{x}$ not on the hypersphere represented by $\sigma$ gives

$$
x \cdot \sigma=x \cdot\left(c-\frac{1}{2} \rho^{2} n_{\infty}\right)=-\frac{1}{2}\left((\mathbf{x}-\mathbf{c})^{2}-\rho^{2}\right)
$$

then $x \cdot \sigma>0$ if the point $\mathrm{x}$ is inside the hypersphere and $x \cdot \sigma<0$ if the point $\mathrm{x}$ is outside the hypersphere. This gives us a way to determine the relative positions of a point and a hypersphere in any dimension.

In these settings a hyperplane $\Pi$ is a hypersphere with a point at infinity, hence $\Pi=p_{1} \wedge \cdots \wedge p_{n} \wedge n_{\infty}$. A Euclidean hyperplane is thus defined with $n$ Euclidean points. It is dually represented by the vector $\Pi^{*}=\pi=\mathbf{n}+\delta n_{\infty}$ where $\mathbf{n}$ is the normal to the hyperplane and $\delta$ the distance to the origin along $\mathbf{n}$. Hence for a point $\mathbf{x}$ of the hyperplane represented by $\pi$ we have

$$
x \cdot \pi=\mathbf{x} \cdot \mathbf{n}-\delta=0
$$

which is the usual (i.e. in linear algebra setting) equation for an hyperplane of normal vector $\mathbf{n}$ and distance $\delta$ to the origin along $\mathbf{n}$. Thus, for a point $\mathbf{x}$ not on the hyperplane, we can determine the relative positions of $\mathbf{x}$ and $\pi$ using the sign of $x \cdot \pi$.

Flats. Flats ( $k$-flats) are offsets of $k$-dimensional subspaces of $\mathbb{R}^{n}$ (i.e. lines, planes, etc). In the CGA framework they are represented by algebra elements of the direct form: $p \wedge \mathbf{A}_{k} \wedge n_{\infty}$, where $\mathbf{A}_{k}$ is a Euclidean blade (i.e. a blade with no vector $n_{o}$ or $n_{\infty}$ as factor) and the vector $p$ represents the Euclidean point $\mathbf{p}$ the flat is passing through. Dualizing this expression leads to the dual form of a $k$-flat: $-p\rfloor\left(\mathbf{A}_{k}^{\star} n_{\infty}\right)$, where $\mathbf{A}_{k}^{\star}=\mathbf{A}_{k} \mathbf{I}_{n}^{-1}$ is the Euclidean dual of $\mathbf{A}_{k}$.

Rounds. Rounds are geometric algebra elements representing $k$-spheres. They can be defined using the outer product of $k+2$ independent vectors representing Euclidean points: $\Sigma=p_{1} \wedge \cdots \wedge p_{k+2}$. 
Hence a circle (i.e a 1-sphere) is defined by three points and a 2-sphere is defined by four points. The minimum number of points to obtain a round is 2 , this corresponds to a 0 -sphere which is a point pair. This is easily explained if we use an alternate definition of a $k$-sphere as the intersection of a hypersphere with a $(k+1)$-flat. So the intersection of a hypersphere with a line gives a 0 -sphere which is the two points of intersection.

In the CGA framework the intersection of an hypersphere $\Sigma^{*}=\sigma$ and a flat $\Pi^{*}=\pi$ in dual form is easily computed by:

$$
\sigma \wedge \pi=\Sigma^{*} \wedge \Pi^{*}
$$

using the formula $5(\Sigma \cap \Pi)^{*}=\Sigma^{*} \wedge \Pi^{*}$ (see [6]). This leads to the dual form of a round with center $\mathbf{c}$ and radius $\rho$ :

$$
\left.\Sigma^{*}=\sigma=\left(c-\frac{1}{2} \rho^{2} n_{\infty}\right) \wedge(-c\rfloor\left((-1)^{(n-k)} \mathbf{A}_{k}^{\star} n_{\infty}\right)\right)
$$

where the change of sign is to maintain coherent orientation. Dualizing this expression leads to another direct form of a round:

$$
\left.\Sigma=\left(c+\frac{1}{2} \rho^{2} n_{\infty}\right) \wedge(-c\rfloor\left((-1)^{k} \mathbf{A}_{k} n_{\infty}\right)\right)
$$

with center $\mathbf{c}$ and radius $\rho$.

Imaginary Hyperspheres and Imaginary Rounds. In this last expression the algebra element $\left(c+\frac{1}{2} \rho^{2} n_{\infty}\right)$ has a particular meaning. For a Euclidean point $\mathbf{x}$, considering the equation $x .\left(c+\frac{1}{2} \rho^{2} n_{\infty}\right)=0$ leads to $(\mathbf{x}-\mathbf{c})^{2}=-\rho^{2}$. This means that the squared distance of all Euclidean points satisfying $x .(c+$ $\left.\frac{1}{2} \rho^{2} n_{\infty}\right)=0$ must be negative. By analogy we say that the vector $\left(c+\frac{1}{2} \rho^{2} n_{\infty}\right)$ represents an imaginary hypersphere.

If such an imaginary hypersphere is used in the definition of the dual form of a round given above, we obtain the dual form of an imaginary round. As only squared distances enter in algebra computations, complex numbers are not needed.

Those elements occur naturally as results of intersections when a real solution does not exist (see figure 10).

\section{Discrete Geometric Primitives}

Basic discrete primitives such as discrete straight lines, discrete hyperplanes and discrete hyperspheres [2114] have been defined as all the discrete points

${ }^{5}$ This formula corresponding to the dual of an intersection, called the plunge, is valid because the union of $\Sigma$ and $\Pi$ is the whole space. Otherwise, the same formula can be used but the dual must be taken wrt. the union of $\Sigma$ and $\Pi$ (more details can be found in [6] chap. 14). 


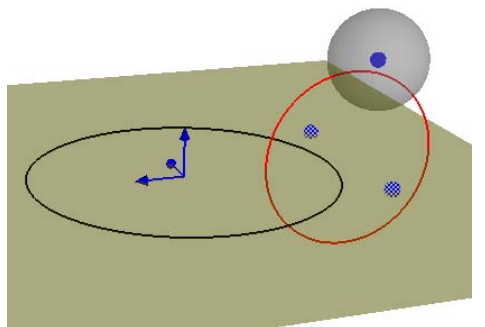

(a) Intersection: imaginary case.

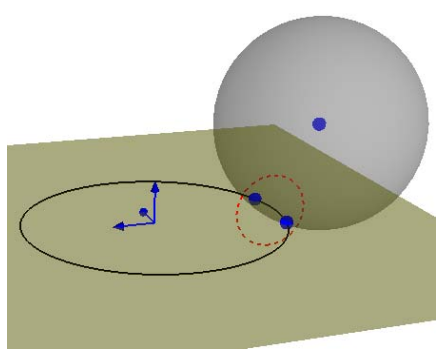

(b) Intersection: real case.

Fig. 1. The intersection of hypersphere $S_{\mathbf{p}, \rho}$ (the sphere) and a 1-sphere $S_{1}$ (the circle). (1a) When there is no intersection the expression $\left.S_{\mathbf{p}, \rho}^{*}\right\rfloor S_{1}$ is an imaginary round (dashed point pair) and the expression $S_{\mathbf{p}, \rho}^{*} \wedge S_{1}^{*}$ is a real round (red circle). (1b) When there is an intersection, real (blue points) and imaginary (dashed circle) are interchanged.

verifying a set of inequalities in the classical linear algebra framework. There is however no direct way to define discrete rounds or flats in such a way. A more recent approach proposed a morphological based digitization scheme [16] defined as the intersection of the discrete space and the Minkowski sum of a structuring element and the object points. For a structuring element corresponding to a ball for a given distance, it is equivalent to considering all the discrete points that are at the ball radius distance of the Euclidean primitive.

For instance in $n \mathrm{D}$ space, considering the ball $B_{2}\left(c, \frac{1}{2}\right)=\left\{x \in \mathbb{R}^{n} \mid d_{2}(x, c) \leq \frac{1}{2}\right\}$ as structuring element, then the discretisation $D(F)$ of a Euclidean object $F$ is defined by:

$$
D(F)=\left(B_{2}\left(x \in F, \frac{1}{2}\right) \oplus F\right) \cap \mathbb{Z}^{n}
$$

where $\oplus$ is the Minkowski sum operator. This can also be interpreted as

$$
D(F)=\left\{X \in \mathbb{Z}^{n} \mid d_{2}(X, F) \leq \frac{1}{2}\right\} .
$$

The problem is to test efficiently the inequality $d_{2}(X, F) \leq 1 / 2$. For instance, let us consider a round $R$ of dimension $k$ defined by

$$
R=\left\{v \in \mathbb{R}^{n}|| c-\left.v\right|^{2}=\rho^{2}\right\} \cap\left\{v \in \mathbb{R}^{n} \mid v=\sum \lambda_{i} u_{i}\right\}
$$

where $\left\{u_{i}\right\}$ are $k$ linearly independent vectors. There is no simple immediate expression for the distance $d_{2}(x, R)$ between a point $x$ and the $k$-dimensional round $R$ in dimension $n$.

In the following subsections, we are going to examine how discrete hyperspheres, hyperplanes, $k$-spheres and $k$-flats can be described within the CGA framework (see also [4]). The interest of those expressions is that they can directly be computed. 


\subsection{Discrete Hyperspheres and Discrete Hyperplanes in CGA}

Using the Euclidean distance, a discrete hypersphere centered at the point $\mathbf{c}$ with radius $\rho$ is defined as:

$$
\left\{\mathbf{p} \in \mathbb{Z}^{n}\left|(\rho-d)^{2} \leq\right| \mathbf{c}-\left.\mathbf{p}\right|^{2}<(\rho+d)^{2}\right\}
$$

where the width $d$ is a positive real number smaller than $\rho$. This is the set of discrete points close to the Euclidean hypersphere.

Hence, a point lies in the discrete hypersphere if it is inside the Euclidean hypersphere of radius $\rho+d$, and outside the hypersphere of radius $\rho-d$.

In the CGA framework these two hyperspheres are defined in dual form as:

$$
\sigma_{\mathbf{c}, \rho+d}=c-\frac{1}{2}(\rho+d)^{2} n_{\infty} \quad \text { and } \quad \sigma_{\mathbf{c}, \rho-d}=c-\frac{1}{2}(\rho-d)^{2} n_{\infty} .
$$

Now, using equation (3) and a discrete point $\mathbf{p} \in \mathbb{Z}^{n}$, distances to the hyperspheres $\sigma_{\mathbf{c}, \rho+d}$ and $\sigma_{\mathbf{c}, \rho-d}$ are checked with the following expressions:

$$
\begin{aligned}
& p \cdot \sigma_{\mathbf{c}, \rho+d}=\frac{1}{2}\left((\rho+d)^{2}-(\mathbf{p}-\mathbf{c})^{2}\right) \\
& p \cdot \sigma_{\mathbf{c}, \rho-d}=\frac{1}{2}\left((\rho-d)^{2}-(\mathbf{p}-\mathbf{c})^{2}\right)
\end{aligned}
$$

expression (4) must be positive and expression (5) must be negative.

Hence, in any dimension, a discrete hypersphere centered at $\mathbf{c}$ with radius $\rho$ is defined as:

$$
\left\{\mathbf{p} \in \mathbb{Z}^{n} \mid p \cdot \sigma_{\mathbf{c}, \rho-d}<0 \text { and } p \cdot \sigma_{\mathbf{c}, \rho+d} \geq 0\right\} .
$$

The figure 2 shows an example of a discrete hypersphere drawn with this definition.

Using the same development, a discrete hyperplane can be defined as the set of discrete points close to the Euclidean hyperplane $\pi_{\mathbf{n}, \delta}=\mathbf{n}+\delta n_{\infty}$ and we must find the discrete points enclosed between two hyperplanes. To do this, we define two hyperplanes $\pi_{\mathbf{n}, \delta-d}=\mathbf{n}+(\delta-d) n_{\infty}$ and $\pi_{\mathbf{n}, \delta+d}=\mathbf{n}+(\delta+d) n_{\infty}$ translated for

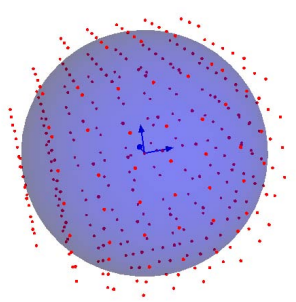

(a)

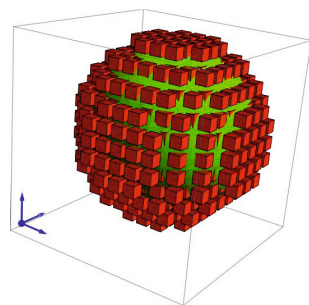

(b)

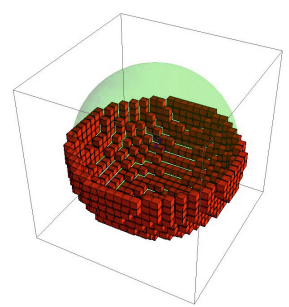

(c)

Fig. 2. Example of a discrete hypersphere. (2a) Drawn with the "centers" of the voxels. (2b) Drawn with voxels. (2c) Partial view. 
a width $d$ along the normal of the first one. Hence, in any dimension, a discrete hyperplane with normal $\mathbf{n}$ at distance $\delta$ to the origin along $\mathbf{n}$ is defined as:

$$
\left\{\mathbf{p} \in \mathbb{Z}^{n} \mid p \cdot \pi_{\mathbf{n}, \delta-d}<0 \text { and } p \cdot \pi_{\mathbf{n}, \delta+d} \geq 0\right\}
$$

which is basically the same definition as for the discrete hypersphere.

To conclude this section, in the CGA framework discrete hyperspheres and discrete hyperplanes are the same objects, and their definition works in any dimension. It simply consists in checking the signs of two vector dot product expressions.

\subsection{Discrete Rounds and Discrete Flats in CGA}

In this section we define discrete rounds (i.e. discrete $k$-spheres) using the structuring element approach. In our case, we use a hypersphere as structuring element. So, we have to check if a given point $\mathbf{p}$ lies into the discrete round by verifying that the hypersphere centered on $\mathbf{p}$ intersects the $k$-sphere.

Two cases must be distinguished depending on the form of the given round to digitize. If it is in direct form, we use an expression involving the left contraction product. Otherwise, if the round is in dual form, an expression with the outer product is used. In both cases, it is easier to consider the hypersphere in its dual form. This situation is usual in the CGA framework as duality is fully integrated. Once we have the expressions, either mode (direct or dual) is easy to work with, it just depends on the way the data have been given.

Rounds in Direct From. Let $S_{k}$ be a $k$-sphere and $S_{\mathbf{p}, \rho}$ a hypersphere with center $\mathbf{p}$ and radius $\rho$. Let $\Sigma_{k}$ be a round in direct form representing $S_{k}$. The intersection of $S_{k}$ and $S_{\mathbf{p}, \rho}$ is given by the formula $\left.S_{\mathbf{p}, \rho}^{*}\right\rfloor \Sigma_{k}$ where $S_{\mathbf{p}, \rho}^{*}$ is the algebra element representing $S_{\mathbf{p}, \rho}$ in dual form (see [6]).

Hence, as $S_{\mathbf{p}, \rho}^{*}$ is a blade of grade 1 and $\Sigma_{k}$ is a blade of grade $k$, the intersection must be a blade of grade $k-1$. This is coherent with the usual result when intersecting a $k$-sphere with a hypersphere we obtain a $(k-1)$-sphere.

Moreover, if no intersection exists, the obtained $(k-1)$-sphere is an imaginary round (see figure 1).

For a round $\Sigma_{k}$ in direct form, its squared radius is given by the formula

$$
\rho^{2}=(-1)^{k} \frac{\Sigma_{k}^{2}}{\left.\left(n_{\infty}\right\rfloor \Sigma_{k}\right)^{2}} .
$$

So, to test if a discrete point $\mathbf{p} \in \mathbb{Z}^{n}$ is in a discrete round we only have to test the sign of the expression

$$
\left.(-1)^{k-1}\left(\left(p-\frac{1}{2} \rho^{2} n_{\infty}\right)\right\rfloor \Sigma_{k}\right)^{2} .
$$

This gives us the definition of a discrete round in direct form

$$
\left.\left\{\mathbf{p} \in \mathbb{Z}^{n} \mid(-1)^{k-1}\left(\left(p-\frac{1}{2} \rho^{2} n_{\infty}\right)\right\rfloor \Sigma_{k}\right)^{2} \geq 0\right\} .
$$


Rounds in Dual Form. Let $S_{k}$ be a $k$-sphere and $S_{\mathbf{p}, \rho}$ an hypersphere with center $\mathbf{p}$ and radius $\rho$. Let $\sigma_{k}$ be a round in dual form representing $S_{k}$. The dual of the intersection of $\sigma_{k}$ and $S_{\mathbf{p}, \rho}$ is given by the formula $\left(S_{k} \cap S_{\mathbf{p}, \rho}\right)^{*}=S_{\mathbf{p}, \rho}^{*} \wedge \sigma_{k}$ where $S_{\mathbf{p}, \rho}^{*}$ is the algebra element representing $S_{\mathbf{p}, \rho}$ in dual form (see [6]).

As before, $S_{\mathbf{p}, \rho}^{*}$ is a blade of grade 1 and $\sigma_{k}$ is a blade of grade $n+2-k$ thus the dual of the intersection is a blade of grade $n+3-k$ so its dual is a blade of grade $(k-1)$. Expression $S_{\mathbf{p}, \rho}^{*} \wedge \sigma_{k}$ corresponds to a $(k-1)$-sphere represented by a round in dual form.

The squared radius of a round $\sigma_{k}$ in dual form is given by the formula

$$
\rho^{2}=(-1)^{n+1-k} \frac{\sigma_{k}^{2}}{\left.\left(n_{\infty}\right\rfloor \sigma_{k}\right)^{2}} .
$$

So, to test if a discrete point $\mathbf{p} \in \mathbb{Z}^{n}$ is in a discrete round we only have to test the sign of the expression

$$
(-1)^{n-k}\left(\left(p-\frac{1}{2} \rho^{2} n_{\infty}\right) \wedge \sigma_{k}\right)^{2}
$$

This gives us the definition of a discrete round in dual form

$$
\left\{\mathbf{p} \in \mathbb{Z}^{n} \mid(-1)^{n-k}\left(\left(p-\frac{1}{2} \rho^{2} n_{\infty}\right) \wedge \sigma_{k}\right)^{2} \geq 0\right\}
$$

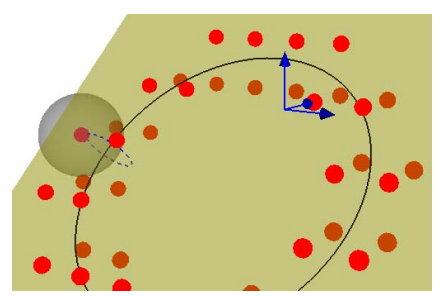

(a)

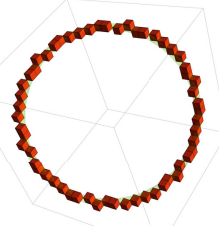

(b)

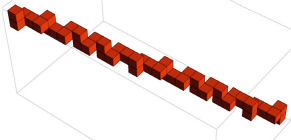

(c)

Fig. 3. Examples of discrete $k$-spheres. (3a) Using a hypersphere as structuring element. The points are the "centers" of the voxels defining a discrete circle. (3b) A discrete circle. (3c) A discrete line generated in the same way as the discrete circle.

To conclude this section about discrete rounds, we have seen that discrete rounds can be defined from rounds either in direct form or dual form. The structure of the definitions is the same, the only difference is in the involved product.

Moreover, as $k$-flats can be seen as particular rounds passing through infinity, from those definitions, discrete circles, lines and so on can easily be defined in any dimension. 


\section{Conclusion and Future Works}

In this paper, definitions of discrete hyperspheres, hyperplanes and rounds (i.e. circles, lines, spheres, flats in any dimension) have been proposed in the Conformal Geometric Algebra formalism. These definitions are valid in any dimension. The expressions are simple and can be directly used in computations contrary to equivalent definitions in a classical framework. They also propose a unified approach for such discrete objects as $k$-flats are a special case of $k$-spheres. One of the hopes, beyond the definitions in dimension $n$, is that generation and recognition algorithms of $k$-spheres and flats can be somewhat unified in a more general framework.

However, efficient implementation of the Conformal Geometric Algebra is not an easy task 611139] and future work is needed to have a specialized implementation for discrete geometry. For this article, we have used GAviewer [8] and the Mathematica package [3].

First experimentation has been conducted about discrete rotations. In Conformal Geometric Algebra, plane rotations are defined in a simple way as:

$$
R=\mathbf{b a}=\mathbf{b} \cdot \mathbf{a}+\mathbf{b} \wedge \mathbf{a}=\cos (\phi / 2)-\sin (\phi / 2) \mathbf{I}
$$

where $\mathbf{a}$ and $\mathbf{b}$ are two purely Euclidean vectors (i.e. without $n_{o}$ and $n_{\infty}$ ) $\phi$ is the rotation angle (i.e. $\phi / 2$ is the angle from $\mathbf{a}$ to $\mathbf{b}$ ) and $\mathbf{I}$ is the unit bivector for the plane $\mathbf{a} \wedge \mathbf{b}$ (i.e. $\mathbf{I}$ is such that $\mathbf{a} \wedge \mathbf{b}=\beta \mathbf{I}$ with $\beta>0$ ). Then $R p R^{-1}$ is the rotation of a point represented by the vector $p$ and $R^{-1}=\cos (\phi / 2)+$ $\sin (\phi / 2) \mathbf{I}$.

If one wants to define a plane rotation using only integer numbers, we use the expression

$$
R=\alpha-\beta \mathbf{I}
$$

where $\alpha$ and $\beta$ are integer numbers. This corresponds to the use of two vectors $\mathbf{a}^{\prime}$ and $\mathbf{b}^{\prime}$ with angle $\phi / 2$ but with integer coordinates. In that case a coefficient appears in the expression of $R^{-1}$ because $R$ is not of norm 1 . Thus

$$
R^{-1}=\frac{1}{\alpha^{2}+\beta^{2}}(\alpha+\beta \mathbf{I})
$$

Now, if the vector $p$ represents a point $\mathbf{p}$ with integer coordinates, its rotation is computed by $\left(\alpha^{2}+\beta^{2}\right) R p R^{-1}$. In dimension 2 this means that we have to consider the rotation as a function from $\mathbb{Z}^{2}$ to $\mathbb{Z}^{2}$ and define a grid $\left(\alpha^{2}+\beta^{2}\right)$ times smaller for the image space. Such phenomenon provides a good explanation of why a discrete rotation is not a one-to-one application. Further investigations need to be conducted taking into account not only rotations but also translations in order to be able to handle rigid transforms for example. 


\section{References}

1. Andres, E., Acharya, R., Sibata, C.: Discrete analytical hyperplanes. Graphical Models and Image Processing 59(5), 302-309 (1997)

2. Andres, E., Jacob, M.-A.: The discrete analytical hyperspheres. IEEE Transactions on Visualization and Computer Graphics 3(1), 75-86 (1997)

3. Aragon-Camarasa, G., Aragon-Gonzalez, G., Aragon, J.L., Rodriguez-Andrade, M.A.: Clifford Algebra with Mathematica. ArXiv e-prints (October 2008)

4. Aveneau, L., Andres, E., Mora, F.: Expressing discrete geometry using the conformal model. Presented at AGACSE 2012, La Rochelle, France (2012), http://hal.archives-ouvertes.fr/hal-00865103

5. Bayro-Corrochano, E., Scheuermann, G. (eds.): Geometric Algebra Computing in Engineering and Computer Science. Springer (2010)

6. Dorst, L., Fontijne, D., Mann, S.: Geometric Algebra for Computer Science: An Object Oriented Approach to Geometry. Morgan Kauffmann Publishers (2007)

7. Dorst, L., Lasenby, J. (eds.): Guide to Geometric Algebra in Practice (Proceedings of AGACSE 2010). Springer (2011)

8. Fontijne, D., Dorst, L., Bouma, T., Mann, S.: GAviewer, interactive visualization software for geometric algebra (2010), Downloadable at

http://www.geometricalgebra.net/downloads.html

9. Fuchs, L., Théry, L.: Implementing geometric algebra products with binary trees. Advances in Applied Clifford Algebra (published online first, 2014), http://dx.doi.org/10.1007/s00006-014-0447-3

10. Hestenes, D.: New Foundations for Classical Mechanics. D. Reidel Publ. Co., Dordrecht (1986)

11. Hildenbrand, D.: Foundations of Geometric Algebra Computing. Geometry and Computing, vol. 8. Springer (2013)

12. McDonald, A.: A survey of geometric algebra and geometric calculus (2013), http://faculty.luther.edu/ macdonal/index.html\#GA\&GC (last checked February 2014)

13. Perwass, C.: Geometric Algebra with Applications in Engineering. Springer (2009)

14. Reveillès, J.-P.: Géométrie Discrète, calculs en nombres entiers et algorithmique. Thèse d'état, Université Louis Pasteur, Strasbourg, France (1991)

15. Sommer, G.: Geometric computing with Clifford algebras: theoretical foundations and applications in computer vision and robotics. Springer (2001)

16. Tajine, M., Ronse, C.: Hausdorff discretizations of algebraic sets and diophantine sets. In: Nyström, I., Sanniti di Baja, G., Borgefors, G. (eds.) DGCI 2000. LNCS, vol. 1953, pp. 99-110. Springer, Heidelberg (2000)

17. Wareham, R., Cameron, J., Lasenby, J.: Applications of conformal geometric algebra in computer vision and graphics. In: Li, H., J. Olver, P., Sommer, G. (eds.) IWMM-GIAE 2004. LNCS, vol. 3519, pp. 329-349. Springer, Heidelberg (2005) 